\title{
Modeling the Contributory effect of Impairment factors on Voice Transmitted over the Internet
}

\author{
Adegbenro $\mathrm{O}$. \\ Department of \\ Electrical \\ and Electronics \\ Engineering, \\ Covenant University \\ of Lagos, \\ Lagos State, \\ Nigeria
}

\author{
John S.N. \\ Department of \\ Electrical \\ and Information \\ Engineering \\ Covenant University, \\ Ota, \\ Ogun State, Nigeria
}

\author{
NdujiubaC. \\ Department of \\ Electrical \\ and Information \\ Engineering \\ Covenant University, \\ Ota, \\ Ogun State, Nigeria
}

\author{
Akinade B. A. \\ Department of \\ Electrical \\ and Electronics \\ Engineering, \\ Covenant University \\ of Lagos, \\ Lagos State, Nigeria
}

\begin{abstract}
The emergence of VoIP in the growing Internet services today has brought about new way in which services are rendered to the public. In viewofprovidingbetter alternative to Public Switch Telephone Network (PSTN) with high quality and efficient internet telephony (VoIP), there is a need of having an improved QoS in Voice transmitted over the Internet.In this work, the influence of different impairment factors on Voice over Internet Protocol (VoIP) as a contributory factor were analyzed, characterized, and evaluated. The results obtained from the research work compared with the theoretical values have some common correlations. This indicated that the impairment factors have significant contributory effect on voice transmitted over the internet.
\end{abstract}

\section{Key Words}

E-Model, Quality of Service, Mean Opinion Score, Impairment Factors, ContributoryEffects, Voice-over-IP

\section{INTRODUCTION}

VoIP is flexible in providing new services and its cost saving advantage as a real-time application but shrouded with challenges that demands attention for toll quality [1]. More importantly some customers carry out bulk of their businesses over the phone requiring minimal impairment for excellent quality that is essential to their success.Based on the above background, in this study a subjective network performance metric Model (E-Model) is being developed and used to estimate relative quality of the E-Model outputwith impairment factors. The different impairment factors are latency (i.e. end-end-delay), packet loss, jitter and equipment used in the network which actively affect the VoIP performance index [3], [4]. The result obtained from an experimental test bed gives the performance metric index expressed as Mean Opinion Score (MOS) and RFactor - the baseson how good the transmitted voice quality are measured.The MOS ranges from 0 to 5 in their measurement values of which "zero"indicates a very poor grade, while the factor of "five"represents an excellent voice quality. The Rfactor, which ranges from 0 to100, defines the quality of the voiceobtainable from MOS through a linear mapping [5].This is an empirical approach for estimating satisfactory level of voice transmitted over the internet, a value above 70 for medium quality and the degree of voice fidelity decreases as the scale goes down. The objective of call quality is toestimate the impact of latency and its accompanying voice degrading factors to obtain reliable estimate using subjective testing method as an example (E-Model) [6].

In general, voice inVoIP is degraded by impairment factors as mentioned aboveit isobserved that latency contributes the greatest delay in the context of the impairment factors affecting quality of service in VoIP network. The results obtained from G.711 and G.729 (the equipment used for the experiment), revealed that in a complex network with QoS enabled environment, the MOS maintained constant rating values over some packets delay while the R-factor varies. This indicates that, $\mathrm{R}$-factor has a better performance metric in estimating VoIP Quality.Simulation and experimental results obtained using codec equipment were compared to those obtained using Telchemy IP Impairment Simulator software $\odot$ [7]. The voice qualities were affected by codecs, delay, and packet loss which are (the impairment factors). Sources of such impairments are network performance, routing path of calls and the configuration of network equipment. Based on these outlined factors, there is a need to perform an experiment on the network performance toward improving the QoS in the VoIP and its contributory effect on voice transmitted over the internet.

This work is organized as follows. Section two describes VoIP performance model. Section threehighlighted: voice quality standards,protocols and quality of service mechanisms. Sections fourand five analyze the performance metricand methodologies usedin this work.Finally, simulation results and analysis of the experimentwere presented with the conclusionin section sixandseven respectively.

\section{VOIP PERFORMANCE MODEL}

The VoIP performance model for voice transmitted over internet as shown in Figurelis the communication network channel established between two or more LANs along WAN connected with multiple nodes transferring voice packets along the network medium. The server contains the Solar Wind software $\odot$ [8] that enable the measurement of the impairment factors. It is noted that the codec used were of the commonly recommended for voice communicating over the internet. The likes of G.711 and G.729 as shown in Table 1 in section 6.2, help in adding high compressing rate for better voice packet delivery from source to destination. 


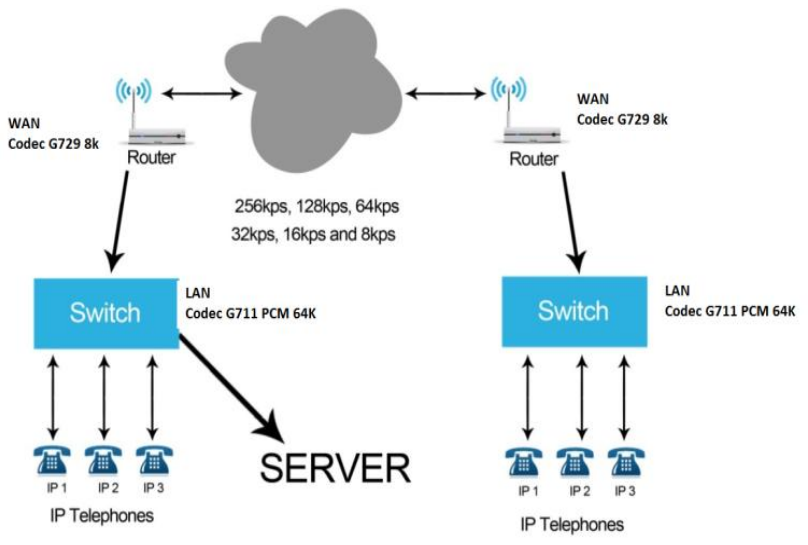

Figure 1.VoIP Performance Model for voice transmitted over the internet.

In this work, a communication model and the scenario presented were real life and experimental network environment using Telechemy simulation software an open source platform [9] and Bandwidth Calculator for evaluation.Also, the tool was used in analysis and evaluating the impairment factors of the voice transmitted over the internet (VoIP) taken in consideration the voice quality standards and protocols.

\section{VOICE QUALITY STANDARDS AND PROTOCOLS}

Traditionally high fidelity remains the quality experience of legacy telephone in communication services. QoS enabled environment require voice quality standards and protocols in place. The standards and protocols used in VoIP include: Internet Engineering Task Force (IETF), International Telecommunication Telephone union(ITU), which implements voice transmission functionsand include at least one voice codec that sends and receives packetized voice, Real Time Transport Protocol (RTP) that provides the transport for carrying audio/media portion of VoIP communication and Session Initiation Protocol (SIP) -an IETF standard protocol that initiate user sessions and enables users to initiate and receive services and communications from any location. Also call transfer and termination are established by session initiation protocol [3], [10], [11],[15].

\subsection{QoS Mechanisms in VoIP}

Implementing QoS mechanisms is another key consideration in order to ensure that VoIP traffic is forwarded across a network in a timely manner. A variety of different queuing mechanisms are used on WAN interfaces to help prioritize voice traffic in order to ensure that it is serviced in this manner, and not delayed by other traffic that is less time-sensitive. The QoS implementation depends on the coordinated efforts of multiple parties as well, such as: Integrated services (InterServ), reservation protocol (RSVP), differentiated Services (DiffServ) and multiprotocol Label Switching (MPLS).[3], [11], [12],[13], [14].

\section{PERFORMANCE METRICS OF VOIP}

Voice over IP technology offers a wide range of benefits which include: reduction of telecom costs, management of one network instead of two, simplified provisioning of services to remote locations, and the ability to deploy a new generation of converged applications. But no business can afford to have its voice services compromised. Revenue, relationships and reputation all depend on people being able to speak to each other on the phone with high availability on a given computer system.
Thus, every company pursuing the benefits of VoIP must take steps to ensure that their converged network delivers acceptable call quality and non-stop availability

This behavioural pattern is influenced by Performance metric [16], [17], otherwise known as the impairment factorsin voice transmitted over the internet as illustrated in Figure 2.

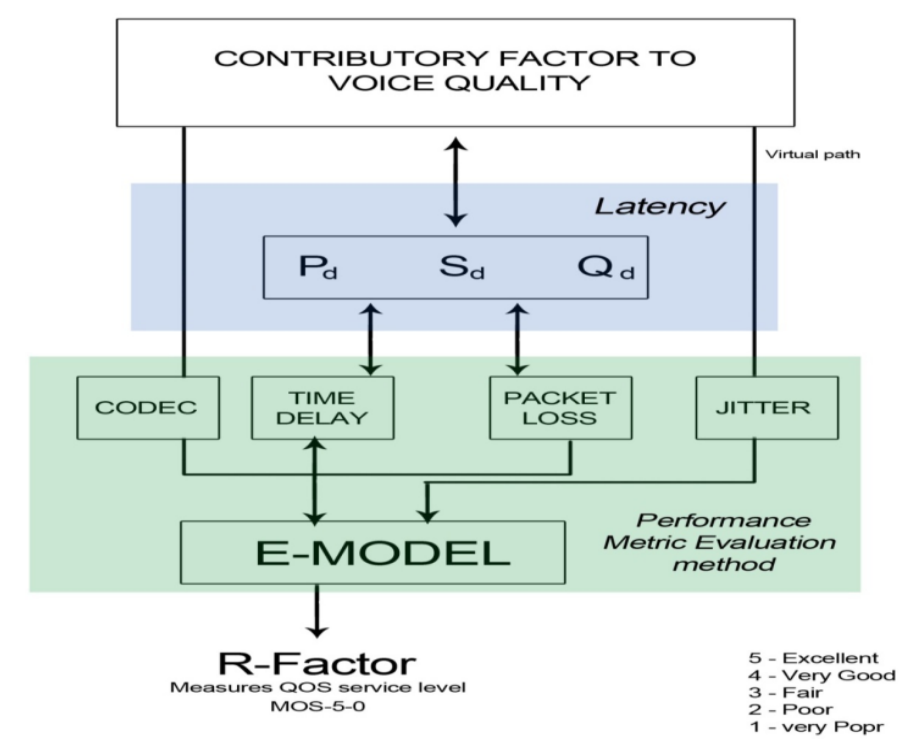

Figure 2.Functional Diagram of Impairment factors in voice transmitted over the internet.

The VoIP performance metrics impairment includes delay, jitter, packet loss and Mean Opinion Score (MOS) [18], [19]. End-toend delay (latency) is the time it takes voice packets to traverse from source to destination through the channel. Latency is afunction of delay caused by propagation, serialization and Queuing that degraded voice quality.Thepacket to packet delay caused by queuing and congestion, leads to variable delay known as jitter in packets' arrival time.Packet loss stands as a problem in the networks and when it attains a certain threshold, it results to voice degradation.Codec is the equipment used for compression and decompression of packets which add further delay that impairs voice quality [1], [20]. Codecs like G.711, G.729 used in this work and G.723 compress voice from 64kbps to a certain bit rate such as $8,6,4$, and at times $5.3 \mathrm{kbps}$ for audio.

The delay in VoIP network is kept below 250ms for real-time conversations of to and froconnection and is the recommended acceptable delay threshold by ITU-T in toll quality of voice transmitted over the internet.Likewise the jitter experience is taken as $30 \mathrm{~ms}$ and the packet loss real-timemeasurement is about $5 \%$ or a little lower [11].The MOS also as a subjective metric estimates the user satisfaction by means of a score whichranges from 1.0 (poor) to 5.0 (good) in performance evaluation [1], [6], [20].Hence, the performance metric of VoIP can be viewed in terms of its effective throughput of the voice transmitted over the network/internet. Throughput shows the quality of error-free data/voice that is transmitted over a communication channel per unit time. Therefore,

$$
\text { Throughput }=\frac{\text { Bandwidth }}{\text { TotalLatensy }}, \text { bps }
$$


also,

$$
\text { Thoughput }=\frac{M S S}{R T T} * \frac{1}{\sqrt{p}}, \text { bps }
$$

where, MSS - Maximum segment size (fixed for each internet path, typically 60 bytes)

RTT - Round trip time (as measured by TCP) and P - Packet loss rate (\%) [21]. In summary, the parameters acceptable levelfor VoIP performance thresholds are Delay $\leq 250 \mathrm{~ms}$; jitter $\leq 30 \mathrm{~ms} ; \quad$ packet Loss $\leq 5 \%$ andMOS $\geq 3$. This is achievable with use of codecs.

\section{METHODOLOGY}

In this work, empirical approach using impairment factors (latency, packet delay and equipment used) in E-Model was used to study the contributory effect of latency on voice transmitted over the internet in VoIP network. This was achieved from the concept of Performance metric by non-linear mapping of Mean opinion Score (MOS) to estimate relative quality of voice over the internet. Solar wind Software $\odot$ and Telechemy simulation $(\subset$ tools in a quality of service (QoS) enabled environment were used for this experiment.

The E-model as one of the toolsused in estimating voice quality in communication systemis a subjective model based on additive principle for evaluating the total relative voice quality in the presence of impairment factors.Generally,the performance metric index expressed as R-Factor hasa mathematical expression as shown in equation 1 . The mean opinion score helps to pinpoint any specific places or times where voice quality is unacceptable and R-factor defines the quality of the voice obtainable from MOS through mapping. The expression of the mapping is shown as follows:

$R=R_{o}-I_{s}-I_{d}-I_{e}+A$

where, Ro = Basic signal-to-Noise-Ratio (SNR)

$I_{s}=$ combination of all impairments which occur simultaneously with voice signal

$I_{d}=$ impairment due to delay

$I_{e}=$ impairment caused by low bit rate codec (equipment)

$A=$ advantage factor (attempts to account for caller expectation)0 for wire lineand 10 for GSM [24], [25].Among all the values in equation[1], only $I_{d}$ and $I_{e}$ are typically considered variable in aVoIP system. Using default values for all other factors reduces model to:

$R=93.4-I_{d}-I_{e}$ (codecloss)

The total delay, $I_{d}$, which comes from the backlog of the systems during data/voice performancewithin the channel from the experiment performed in the course of the research work gives the following mathematical equation and expressed as follows: $I_{d}$ $=T_{\text {total }}=T_{A}+T_{D} ;$ where, $T_{A}=$ Encoding time, $T_{D}=$ Delay; but $T_{D=} T_{p}+T_{S}+T_{q}$

Where, $T_{p}$ - time of propagation, $T_{s}-$ the serialization time, $T_{q}-$ the queuing time.

Hence:

$$
T_{\text {total }}=T_{A}+T_{D} \in\left(T_{p}+T_{S}+T_{q}\right)
$$

As a result, latency from equation 3 which is the summation of delays inherent on the network can be derived into the follows [2]:

$$
T_{p}+T_{s}+T_{q}=\frac{x}{0.667 c}+\frac{N \mu}{(\mu-\lambda) B}+\frac{\lambda^{2}}{(1-\rho) B \mu^{2}},
$$

Substituting equation 4 into equation [1] becomes:

$$
\mathrm{R}=93.4-\frac{\sum x_{n}}{0.667 c}+\frac{\sum N_{n} \mu}{\left(\mu-\lambda_{n}\right) B}+\frac{\sum \lambda_{n}^{2}}{\left(\mu-\lambda_{n}\right) B \mu}-\mathrm{I}_{\mathrm{e}}
$$

where: $I_{d}=$ impairment due to delay $=$

$$
\frac{\sum x_{n}}{0.667 c}+\frac{\sum N_{n} \mu}{\left(\mu-\lambda_{n}\right) B}+\frac{\sum \lambda_{n}^{2}}{\left(\mu-\lambda_{n}\right) B \mu}
$$

The E-Model, as described in ITU-T G.107, presents informative computational model for predictive analysis

\section{SIMULATION RESULTS AND ANALYSIS OF THE EXPERIMENT}

Based on the model for this work in Figure 1, Telechemy VoIP Simulator experimental setup was used as a test-bed to estimate the performance metric of voice over internet protocol transmitted on network. The experimental setup consists of a Local Area Network (LAN) and a remote network (WAN) with the transmission carried out varying by the number of users, bandwidth, maximum transmission unit (MTU) in a QoS enabled environment. G.711 and G.729 codec were used differently to compare their performance in the network. The objective here is to compare the degree of impairment caused by latency in the overall voice quality estimation at the receiving end.Results obtain from the various experiments performances areshown in Figure 4 and Figure 5.

\subsection{Experimental Result Using Telechemy Simulator}

Using Telechemy simulator, the number of occupancy were varied from 5 to 20 on both sites of the system (Local LAN and at the remote LAN) using codec G.711 and G.729 in each case. The performance Metric Evaluation Experiment(Table 1) shows the experimental result obtained using the following parameters: the bandwidth $-256 \mathrm{kbps}$, the MTU $=1500$ bytes and QoS enabler mechanism. Figure 3 shows the result obtained using codec G.729, and the graph result gives the average jitter as $2.8 \mathrm{~ms}$ while the packet loss stands at $0.34 \%$.
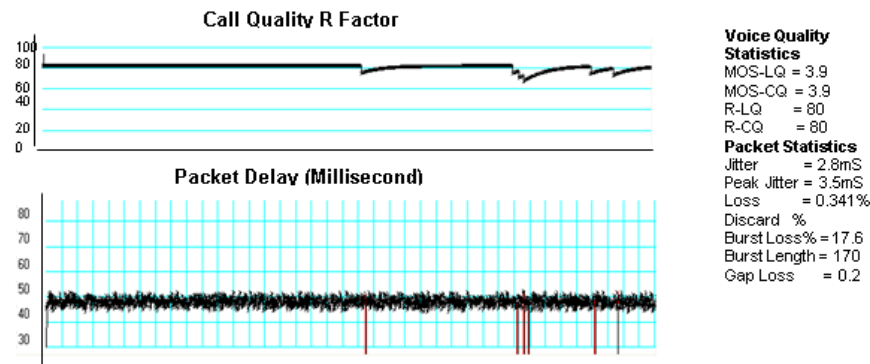

Figure 3: Simulation result of R-factor and Packet delay using G. 729 PLC Codec. Workload = 10, Bandwidth = 256kbps, MTU=1500bytesand QoS enabled. 
Similarly using the same parameters with G.711 Codec in Figure 4 , the following results were obtained: toll quality of 4.2 for both MOS (LQ) and (CQ), and R-factor of 90. The average jitter obtained was $1.5 \mathrm{~ms}$ and the packet loss of $1.07 \%$. Comparing the results obtained during the experiment, we observed that the impairment factor of the voice quality achieved was better when using G.711 compared to G.729 as shown in Figure 4.

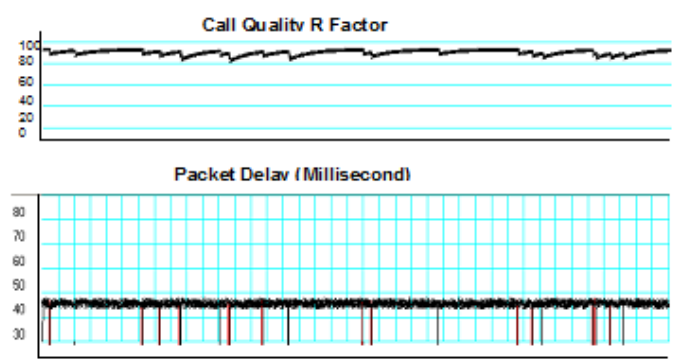

Figure 4: Simulation result of R-factor and Packet delay using G 711 PLC Codec. Workload = 10, Bandwidth = 256kbps, MTU=1500bytes and QoS enabled.

\subsection{Experimental Result Using VoIP Quality and Bandwidth Calculator}

VoIP Quality and Bandwidth Calculator were used to ascertain the performance of MOS, R-factor and packet loss rate from the information transmitted over a communication channel. G.729 was used varying packet loss rate from $0 \%$ to $50 \%$ with constant bandwidth as shown in Table 2 . Figure 5a shows that relative voice quality transmitted over the internet drops as the packet loss rate increases while voice quality improves as the packet loss rate reduces. The Toll quality reached when the packet loss rate at $2 \%$ with R-Factor of 80 . Therefore, the results in Figure 5 a shows that toll quality is achievable between $0 \%$ and $2 \%$ of packet loss rate i.e. $0 \leq$ toll quality $\leq 2 \%$. Similarly from Figure $5 \mathrm{~b}$, when packet loss rate is $4.0 \%$, the MOS becomes 4.2 and when the packet los rate attains $0 \%$ then the MOS becomes 4.4 . Result fromFigure $5 \mathrm{~b}$ shows that: $0 \leq$ toll quality $\leq 4.4$. Figure $5 \mathrm{c}$ is a result from same experiment that validates this work as the toll quality was attained at MOS of 4.4and R-factor of 93.4 which is in agreement with the theoretical value.

Table 1.Performance Metric Evaluation Experiment

\begin{tabular}{|c|c|c|c|c|c|c|c|c|c|}
\hline $\mathrm{Q}_{0 \mathrm{~S}}$ & \multicolumn{9}{|c|}{ Enabled } \\
\hline $\begin{array}{l}\text { Bandwidth } \\
\text { (klps) }\end{array}$ & \multicolumn{9}{|c|}{256} \\
\hline \multirow{2}{*}{$\begin{array}{l}\text { MTU Size } \\
\text { (Kbyte) }\end{array}$} & \multicolumn{9}{|c|}{1.5} \\
\hline & $\begin{array}{l}\text { Work } \\
\text { load }\end{array}$ & $\begin{array}{l}\text { IITU } \\
\text { Size } \\
\text { (KB) }\end{array}$ & $\begin{array}{l}\text { MOS } \\
\text { (LQ) }\end{array}$ & $\begin{array}{l}\mathrm{MOS} \\
(\mathrm{CQ})\end{array}$ & $\begin{array}{c}\text { R-Factor } \\
\text { (LQ) }\end{array}$ & $\begin{array}{c}\text { R-Factor } \\
\text { (CQ) }\end{array}$ & $\begin{array}{c}\text { LOSS } \\
\text { RATE } \\
\%\end{array}$ & $\begin{array}{l}\text { AVERAGE } \\
\text { JITTER }\end{array}$ & CODEC USED \\
\hline Enabled & 20 & 1.5 & 3.3 & 3.3 & 66 & 66 & 4.039 & 1.5 & G.729A (8kbps) \\
\hline Enabled & 15 & 1.5 & 3.5 & 3.5 & 72 & 72 & 2.238 & 1.5 & G.729A (8kbps) \\
\hline Enabled & 10 & 1.5 & 3.9 & 3.9 & 80 & 80 & 0.341 & 2.8 & G.729A (8kbps) \\
\hline Enabled & 5 & 1.5 & 3.9 & 3.9 & 80 & 80 & 0.341 & 1.5 & G.729A (8kbps) \\
\hline Enabled & 2 & 1.5 & 3.9 & 3.9 & 82 & 82 & 0.097 & 1.5 & G.729A (8kbps) \\
\hline Enabled & 20 & 1.5 & 4.0 & 4.0 & 84 & 84 & 3.5 & 1.5 & G.711 (64kbps) \\
\hline Enabled & 15 & 1.5 & 4.1 & 4.1 & 89 & 89 & 1.509 & 1.5 & G.711 (64kbps) \\
\hline Enabled & 10 & 1.5 & 4.2 & 4.2 & 90 & 90 & 1.071 & 1.5 & G.711 (64kbps) \\
\hline Enabled & 5 & 1.5 & 4.2 & 4.2 & 92 & 92 & 0.146 & 1.5 & G.711 (64kbps) \\
\hline Enabled & 2 & 1.5 & 4.2 & 4.2 & 93 & 93 & 0.49 & 1.5 & G.711 (64kbps) \\
\hline
\end{tabular}


Table: 2. VoIP Quality and Bandwidth Calculator experiment.

\begin{tabular}{|c|c|c|c|c|c|c|}
\hline S/N & $\begin{array}{c}\text { Codec } \\
\text { Type }\end{array}$ & $\begin{array}{c}\text { Frame } \\
\text { Size Load } \\
\text { (ms) }\end{array}$ & $\begin{array}{c}\text { Packet } \\
\text { Loss Rate }\end{array}$ & $\begin{array}{c}\text { R- } \\
\text { Factor }\end{array}$ & MOS & $\begin{array}{c}\text { Bandwidth } \\
\text { (MB) }\end{array}$ \\
\hline 1 & G.711 & 5 & 0 & 93 & 4.4 & 131.2 \\
\hline 2 & G.711 & 5 & 1 & 85 & 4.2 & 131.2 \\
\hline 3 & G.711 & 5 & 2 & 77 & 3.9 & 131.2 \\
\hline 4 & G.711 & 5 & 3 & 71 & 3.7 & 131.2 \\
\hline 5 & G.711 & 5 & 4 & 66 & 3.4 & 131.2 \\
\hline 6 & G.711 & 5 & 5 & 62 & 3.2 & 131.2 \\
\hline 7 & G.711 & 5 & 6 & 58 & 3.0 & 131.2 \\
\hline 8 & G.711 & 5 & 7 & 54 & 2.8 & 131.2 \\
\hline 9 & G.711 & 5 & 8 & 51 & 2.6 & 131.2 \\
\hline 10 & G.711 & 5 & 9 & 48 & 2.5 & 131.2 \\
\hline 11 & G.711 & 5 & 10 & 46 & 2.4 & 131.2 \\
\hline 12 & G.711 & 5 & 15 & 36 & 1.9 & 131.2 \\
\hline 13 & G.711 & 5 & 20 & 30 & 1.6 & 131.2 \\
\hline 14 & G.711 & 5 & 25 & 25 & 1.4 & 131.2 \\
\hline 15 & G.711 & 5 & 30 & 22 & 1.3 & 131.2 \\
\hline 16 & G.711 & 5 & 40 & 17 & 1.2 & 131.2 \\
\hline 17 & G.711 & 5 & 50 & 14 & 1.1 & 131.2 \\
\hline
\end{tabular}

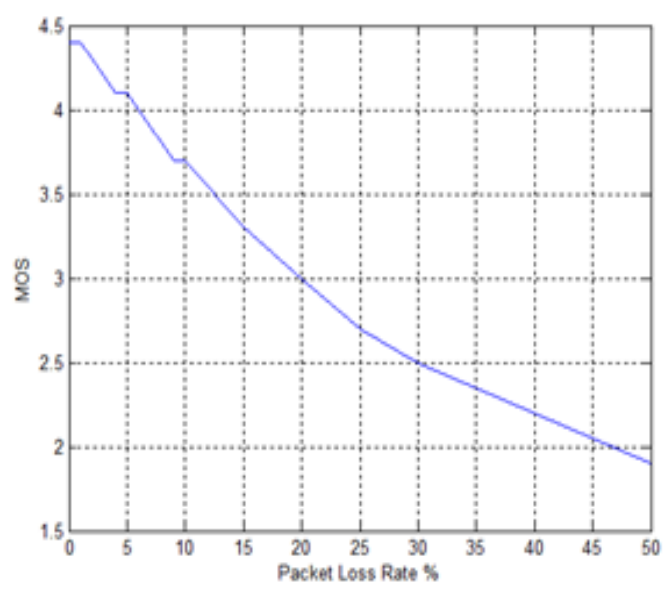

Fig 5a.Graph of R-Factor against Packet Loss

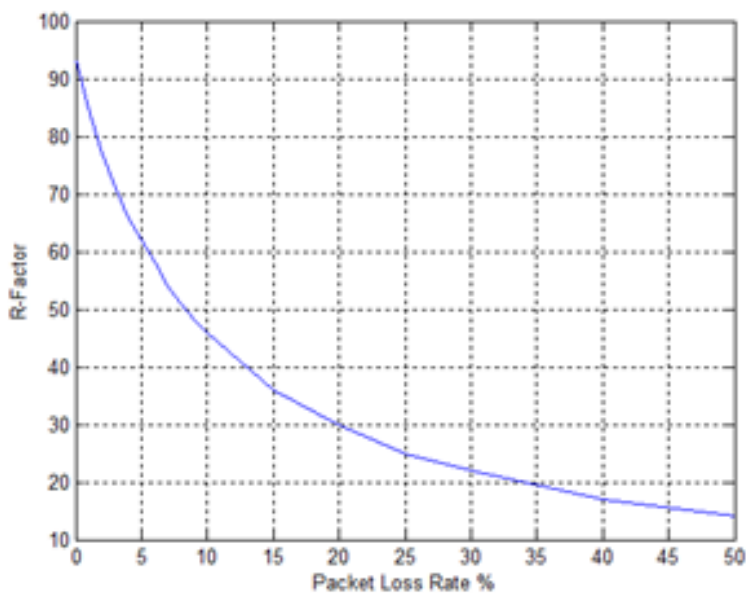

Fig 5b.Graph of MOS against Packet Loss Rate

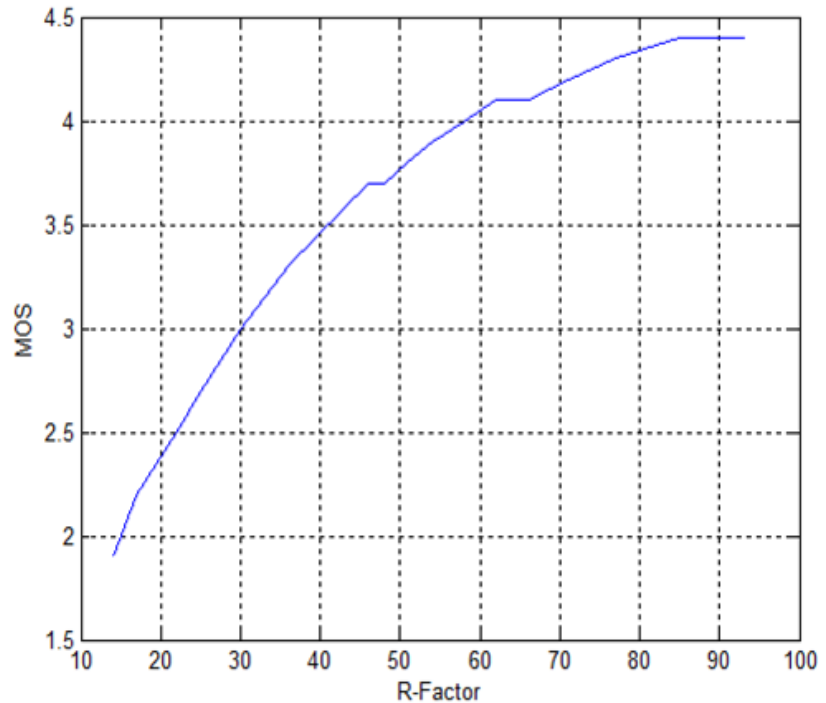

Figure 5c. Graph of MOS against R-Factor

\section{CONCLUSION}

Empirical approach was used in this work with E-Model to characterize the performance of impairment factors in voice transmitted over the internet. These factors i.e latency, packet loss, jitter and efficiency of codec were used with quality of service enabler to estimate relative voice quality in voice network. Furthermore, subjective approach using E-Model to measure the quality of voice in an experiment performed to this effect shows that when Packet loss rate reduces, R-factor and MOS increase in value and toll quality was achieved when packet loss rate lies between $0 \%$ and $2 \%$ i.e $0 \leq$ toll quality $\leq$ $2 \%$. Results obtained by sample measurements from another experiment performedgave acceptable VoIP quality when G.711 and G.729 were used differently in the VoIP network. Also results obtained during the experiment, showed that the impairment factor of voice quality achieved was better when using G.711 compared to G.729 as shown in Figure 4. In order to verify our results a Program was written in $\mathrm{C}$ language with impairment factors to determine the relative voice quality transmitted over the internet.

\section{REFERENCES}

[1] Xianhui Che et al. "VoIP Performance over different Interior Gateway protocols"; International Journal of Communication Networks and Information Security (IJCNIS) Vol. 1, No. 1, April 2009; Pg. 34.

[2] O. Adegbenro et al. "The Contributory Effect of Latency on the Quality of Voice Transmitted over the Internet", Proceedings of The 2011 International Conference on Modeling, Simulation and Visualization Methods" WORLDCOMP '11, July 18-21, 2011, Pg. 278 - 281.

[3] Bur Goode, Senior member IEEE; "Voice over Internet Protocol (VoIP)", Proceeding of the IEEE Vol.90, No. 9, pp 1495-1497, Sept. 2002., 189-207

[4] Zizhi Qiao et al. "A New Algorithm for Speech Quality Improvement in VoIP System" Wireless Peers Commun (2008), October, 2007.

[5] Shu Tao et al, "Improving VoIP Quality Through Path Switching" ; University of Pennsylvania KuaiXu, 
University of Minnesota Antonio Estepa, University of Sevilla...works.bepress.com/lixin_gao/75/; 2005.

[6] Alan Clark "Voice Quality Measurement: Understanding VoIP" CEO and President, 2005

[7] Alan Clark; "Telchemy IP Network Impairment Simulator" version 2.1, 2003

[8] Telechemy, "Voice Quality Measurement", Application Note,March 2008

[9] Kevin Davis, "Understanding Latency in Network Systems: Sources of Latency and Jitter in Network Architectures", White paper by Senior Consultant, NetQoS, 2008, www.netqos.com

[10] David Houck and GopalMeempat, "Call Admission Control and LoadBalancing for Voice over IP," Performance Evaluation 47, (2002) 243-253.

[11] KarieGonia -"Latency and Quality of Service for Voice over IP"; SANS Institute, version 2.4bOption 1,2004.

[12] Henning et al., The Session initiation protocol: Internet Centric Signaling; IEEE Communications Magazine, October, 2000

[13] Xipeng Xiao et al., Internet QoS: A Big Picture"; IEEE Network, March/April, 1999. Pg 8 - 14,.QoS (Quality of Service); www.linktionary.com/q/qos.html

[14] Rajneesh Narula, Kaushal, "Performance Analysis and Evaluation of Hybrid Network using different Integrated Routing Protocols", International Journal Of Computers \& Technology(IJCT), pg. 3090, Dec., 2013.
[15] John Q. Walker et al., "A Handbook for Successful VoIP Deployment: Network Testing, QoS, and More"; NetIQ Corporation; Mission Critical Software for e-business, 2001

[16] Overcoming Barriers to High-Quality Voice over IP Deployments; Dialogic White Paper, Sept. 2007.

[17] Jeffrey Rodman, VoIP to $20 \mathrm{KHz}$ : Codec Choices for High Definition Voice Telephony"; POLYCOM White Paper, July 2008 .

[18] LiotopoulosF.K., Dagiuklas T., V. Karypidou"Vorttex: Evaluation of the perceived VoIP QoS by a third-party, using the E-Model, 2007

[19] John S. N. et al., "Wide Area Network Efficiency Through Optimization of Key Performance Indices"; IEEE Conference, June, 2010.

[20] MiroslawNarbutt et al.,"The Effect of Bandwidth on VoIP Performance in $802.11 \mathrm{~b}$ WLAN Networks", IEE Irish Signals and Systems Conference, Dublin, June 28-30, 2006.

[21] ITU-T Study Group 12, "The E-model, a computational model for use intransmission planning"; ITUTRecommendation G.107, March, 2005.

[22] Ganguly et al., Performance Optimizations for deploying VoIP Services in Mesh Networks; IEEE Journal on Selected Areas in Communications, Vol. 24, N0. 11, Nov. 2006.

[23] S. Tao, K. Xuet al., "Improving VoIP Quality Through Path Switching”, in IEEE INFOCOM, 2005, pp. 2268-2278.

[24] "Voice over IP" (VoIP); SmartBits Performance Analysis System; Spirent Communications, 2001. 